\title{
Vacunación para COVID-19 en niños: el desafío de decidir
}

\author{
COVID-19 vaccine for children: The challenge of making a decision
}

Con el propósito de aliviar y resolver la pandemia de COVID-19, se trabaja actualmente en el desarrollo y estudio de más de 180 vacunas candidatas. ${ }^{1}$ Algunas de ellas se han comenzado a estudiar para su aplicación en niños y adolescentes. $^{2}$ Esta situación lleva a una serie de reflexiones sobre la necesidad y pertinencia de la vacunación para este grupo etario, sobre los aspectos éticos que implican estos estudios, sobre la aceptabilidad de las familias frente a esta posibilidad y sobre nuestra preparación como pediatras para participar y acompañar los procesos de generación de evidencias y juicio crítico de resultados.

Aunque durante los primeros meses de la pandemia el foco de los investigadores estuvo puesto en los ancianos y adultos con determinados factores de riesgo (obesidad, hipertensión, etc.), paulatinamente los niños y adolescentes emergieron como un grupo de interés para la vacunación. Aunque los niños en general podrían ser menos hábiles que los adultos para mantener la distancia física o para la implementación de otras medidas de protección, la evidencia disponible hasta el momento no muestra que sean un grupo de riesgo para enfermedad grave por SARS-CoV-2., ${ }^{3,4}$ Es decir que su posicionamiento como población por vacunar está en función del rol que podrían tener en la transmisión en la comunidad, y por ende, en la protección de la misma. ${ }^{5}$ Además, dada la relativa facilidad de diseminación de esta infección, se estima que es necesaria una cobertura de al menos el $80 \%$ de la población para alcanzar inmunidad de rebaño. ${ }^{6}$ En otras palabras, podría considerarse que hay una necesidad de vacunación relacionada más con el bien común que con el beneficio particular de este grupo etario.

Parte de los fundamentos de esta necesidad se relacionan con el rol de los niños en la diseminación de la infección. Una primera apreciación en ese sentido es que no todos los niños infectados tienen síntomas, pero aun los que tienen, no siempre se diagnostican tempranamente. ${ }^{7}$ Hay datos que muestran que los niños, aun asintomáticos, presentan excreción viral prolongada (de hasta 18 días). Sin embargo, no ha podido establecerse la capacidad infectiva y contagiosidad que ello pudiera implicar. ${ }^{8}$
Para sumar al análisis, los estudios actuales de vacunas (en adultos) no incluyen la medición de la carga viral y excreción viral como un resultado secundario o de transmisión viral, tampoco como dato exploratorio. ${ }^{6}$ Esta falta de información sobre transmisión luego de la vacunación es una oportunidad perdida al momento de pensar la utilidad de la vacuna en la población pediátrica.

Algunos investigadores han ido más allá y se han planteado la posible obligatoriedad de la vacuna. ${ }^{9}$ Han analizado el tema tomando criterios previamente establecidos relacionados con la vacuna, con la enfermedad o con la implementación. ${ }^{10}$ Propusieron como criterio prioritario la seguridad de la vacuna, no solamente por lo que implica per se, sino por el efecto sobre la confianza en las vacunas, en general, en la comunidad. Luego consideraron lo relacionado con la existencia de un subgrupo poblacional muy afectado por la enfermedad, el que los niños tengan un papel en la transmisión (aún no dilucidado) y que la vacuna sea eficaz. ${ }^{9}$ Es claro que antes de plantear esta cuestión hay un paso previo: la investigación.

La investigación tiene dificultades inherentes a la edad de los participantes, en relación directa con su capacidad para consentir. Pero también aspectos éticos ya que, en pos de respetar los principios básicos, solo deberían incluirse en estudios aquellos participantes en quienes el ensayo implique un riesgo mínimo u ofrezca un potencial beneficio que justifique los riesgos. Es decir que debiera contarse con evidencia sólida sobre los aspectos de seguridad, lo que implica tiempo para esperar resultados definitivos de estudios en adultos. Pero también plantea un dilema, ya que esperar hasta establecer inequívocamente seguridad y eficacia en adultos podría retrasar significativamente una respuesta efectiva a la pandemia. ${ }^{1}$

Una alternativa posible es iniciar las investigaciones incluyendo a los fisiológicamente más similares a los adultos: los adolescentes sanos. Ello tiene la ventaja adicional de una mayor capacidad de comprensión de lo que implica la participación en un estudio clínico, aunque sin duda no resuelve lo más desafiante, que es la inclusión de los más chicos y de los que tienen alguna condición de salud que los pone en mayor riesgo de enfermedad grave. 
Superada la etapa de investigación, para que la vacuna llegue a sus destinatarios, debe contarse con aceptabilidad por parte de las familias. Algunos estudios exploraron el tema mostrando, por ejemplo, que, en Estados Unidos, un $65 \%$ (sobre 1500 familias) vacunaría a sus hijos. ${ }^{11}$ Otro estudio mostró que en Inglaterra el $48 \%$ está seguro de querer vacunar a sus hijos. Las inquietudes respecto de la seguridad y eficacia de la vacuna fueron relacionadas con la "rapidez" en su desarrollo. ${ }^{12}$ Cuestiones socioculturales podrían tener relevancia también en este aspecto, por lo que difícilmente estos datos puedan traspolarse a otras comunidades.

Este recorrido, desde laboratorios de investigación básica, implementación de estudios clínicos y políticas de salud pública puede acaso parecer un poco lejano a la tarea cotidiana del pediatra. Sin embargo, los destinatarios de esas iniciativas son nuestros pacientes y sus familias. Es así como los pediatras participamos directa o indirectamente tanto de la atención de niños $y$ adolescentes afectados por esta enfermedad, como de las investigaciones necesarias para conocer de qué se trata la misma y de la educación de la comunidad respecto de qué es y para qué sirve la investigación. Podemos ser parte del equipo de un estudio clínico, y también podemos ser consultados por una familia cuyo hijo/a haya sido invitado a participar en un estudio. Tendremos que evaluar críticamente el resultado de los estudios para definir nuestras indicaciones. Esta situación nos presenta esos desafíos. Nos invita a preguntarnos cuán preparados estamos para decidir lo que consideremos necesario, pertinente, útil y ético para nuestros pacientes.

\section{Dra. María Elina Serra,}

a. FUNDASAMIN-Fundación para la Salud Materno Infantil, CABA, Argentina.

b. Editora asistente, Archivos Argentinos de Pediatría.

Correspondencia:

Dra. María Elina Serra: meserra@fundasamin.org.ar http:/ / dx.doi.org/10.5546/ aap.2021.294

Texto completo en inglés:

http:/ / dx.doi.org/10.5546/aap.2021.eng.294

Cómo citar: Serra ME. Vacunación para COVID-19 en niños: el desafío de decidir. Arch Argent Pediatr 2021;119(5):294-295.

\section{REFERENCIAS}

1. Mintz K, Jardas E, Shah S, Grady C, et al. Enrolling Minors in COVID-19 Vaccine Trials. Pediatrics. 2021; 147(3):e2020040717.

2. Clinical Trials.gov [Acceso: 6 de mayo de 2021]. Disponible en: https: / / clinicaltrials.gov/ ct2/ results?term=vaccine\& cond=Covid19\&age_v $=\&$ age $=0 \&$ gnd $r=\&$ type $=\& r s l t=\& S e$ arch=Apply

3. Rabinowicz S, Leshem E, Pessach IM. COVID-19 in the Pediatric Population-Review and Current Evidence. Curr Infect Dis Rep. 2020; 22(11):29.

4. Zimmermann P, Curtis N. Coronavirus Infections in Children Including COVID-19: An Overview of the Epidemiology, Clinical Features, Diagnosis, Treatment and Prevention Options in Children. Pediatr Infect Dis J. 2020; 39(5):355-68.

5. Kao CM, Orenstein WA, Anderson EJ. The Importance of Advancing Severe Acute Respiratory Syndrome Coronavirus 2 Vaccines in Children. Clin Infect Dis. 2021; 72(3):515-8.

6. Eberhardt CS, Siegrist C-A. Is there a role for childhood vaccination against COVID-19? Pediatr Allergy Immunol. 2021; 32(1):9-16.

7. DeBiasi RL, Delaney M. Symptomatic and Asymptomatic Viral Shedding in Pediatric Patients Infected With Severe Acute Respiratory Syndrome Coronavirus 2(SARS-CoV-2): Under the Surface. JAMA Pediatr. 2021; 175(1):16-8.

8. Han MS, Choi EH, Chang SH, Jin B-L, et al. Clinical Characteristics and Viral RNA Detection in Children With Coronavirus Disease 2019 in the Republic of Korea. JAMA Pediatr. 2021; 175(1):73-80.

9. Opel DJ, Diekema DS, Ross LF. Should We Mandate a COVID-19 Vaccine for Children? JAMA Pediatr. 2021; 175(2):125-6.

10. Opel DJ, Diekema DS, Marcuse EK. A critique of criteria for evaluating vaccines for inclusion in mandatory school immunization programs. Pediatrics. 2008; 122(2):e504-10.

11. Goldman RD, Yan TD, Seiler M, Parra Cotanda C, et al. Caregiver willingness to vaccinate their children against COVID-19: Cross sectional survey. Vaccine. 2020; 38(48):7668-73.

12. Bell S, Clarke R, Mounier-Jack S, Walker JL, Paterson P. Parents' and guardians' views on the acceptability of a future COVID-19 vaccine: A multi-methods study in England. Vaccine. 2020; 38(49):7789-98. 